\title{
Collective excitations in alkali metals on $\mathrm{Al}(111)$
}

\author{
S. R. Barman, ${ }^{1,2}$ C. Stampfl, ${ }^{1}$ P. Häberle, ${ }^{3}$ W. Ibañez, ${ }^{3}$ Y. Q. Cai, ${ }^{1}$ and K. Horn ${ }^{1}$ \\ ${ }^{1}$ Fritz-Haber Institut der Max-Planck-Gesellschaft, D-14195, Berlin, Germany \\ ${ }^{2}$ Inter University Consortium, Khandwa Road, Indore, 452001, M.P., India \\ ${ }^{3}$ Universidad Técnica Federico Santa María, Casilla, Valparaíso, Chile \\ (Received 17 April 2000; revised manuscript received 2 April 2001; published 26 October 2001)
}

\begin{abstract}
The photon-excited multipole plasmon and the bulklike plasmon modes have been studied in $\mathrm{Na} / \mathrm{Al}(111)$ and $\mathrm{K} / \mathrm{Al}(111)$. These collective modes appear for a coverage of $\geqslant 2$ monolayers. Both the multipole and the bulklike plasmon modes exhibit interesting variations in frequency, line shape, and relative intensity with adlayer coverage. The reasons for such behavior are discussed on the basis of existing theoretical results. The substrate $\mathrm{Al}$ multipole plasmon is found to be attenuated by smooth adlayer growth. Deposition of K on two monolayer $\mathrm{Na} / \mathrm{Al}(111)$ results in the growth of $\mathrm{K}$ related collective excitation at the expense of the Na modes. For complicated surface structures like $\mathrm{Al}(111)-(\sqrt{3} \times \sqrt{3}) R 30^{\circ}: \mathrm{K}$ or the $\mathrm{Al}(111)-(2 \times 2)$ : $\mathrm{Na}$ surface alloy, electron charge-density profiles at the surface are calculated based on $a b$ initio density-functional theory-localdensity approximation method to explain the photoyield data. The importance of the shape of the chargedensity profile in determining the collective excitations is demonstrated. For $\operatorname{Al}(111)-(\sqrt{3} \times \sqrt{3}) R 30^{\circ}: \mathrm{K}$, a strong increase in intensity in the energy region of the $\mathrm{K}$ plasmons is observed in the on-top structure which is attributed to a "mixed plasmon" mode. These results show that while the experimental observations for smooth alkali-metal adlayers are in good agreement with existing theory, more theoretical work, especially for the complicated surface structures, is desirable.
\end{abstract}

DOI: 10.1103/PhysRevB.64.195410

PACS number(s): 73.20.Mf, 79.60.Dp

\section{INTRODUCTION}

The investigation of electron collective excitations at metal surfaces is important for an understanding of the response of metals to an incident electromagnetic field. ${ }^{1,2}$ Studies of the collective excitations are important for an interpretation of all surface spectroscopies that use electromagnetic fields or charged particles. It has been recently shown, for example, how surface screening effects can alter the line shape of the direct transition from the $\operatorname{Ag}(111) s$ band. ${ }^{3,4}$ This shows that surface screening effects can influence the spectral line shape in angle-resolved photoemission. A strong modification of the photoemission line shape has been found in Li near its bulk plasmon frequency. ${ }^{5}$ However, direct experimental evidence for the photon excited collective modes on metal surfaces are few, ${ }^{6-16}$ and with only a few exceptions have dealt with clean surfaces, leaving out the interesting field of thin-metal films and adatom-induced surface structures. Monin and Boutry ${ }^{12}$ and Walldén ${ }^{13}$ studied the photoyield in alkali-metal films, but only a few have dealt with a detailed investigation of the emergence of the bulklike and multipole plasmons. Kim et al. ${ }^{14}$ studied K layers using electron-energy-loss spectroscopy (EELS) and total photoyield and observed a peak at $0.8 \omega_{p}$ which was attributed to the multipole plasmon. Barman et al. ${ }^{15}$ identified the multipole plasmon in $\mathrm{Li}$ adlayers, where the bulklike excitation is strongly dampened by the lattice potential. The collective excitations on the $\mathrm{Al}(100)-c(2 \times 2): \mathrm{Na}$ surface alloy phase was studied recently by Barman et al. ${ }^{16}$

The discovery of surface plasmons by Ritche in 1957 (Ref. 17) marked the beginning of the exciting field of plasmon excitations on surfaces. ${ }^{18}$ Using the Maxwell boundary conditions at a sharp metal-vacuum interface, the condition for the existence of the surface plasmon turns out to be $\epsilon=-1$. Assuming the Drude dielectric response

$$
\epsilon(\omega)=1-\frac{\omega_{p}^{2}}{\omega^{2}}
$$

the frequency of the surface plasmon $\left(\omega_{s}\right)$ is given by $\omega_{s}$ $=\omega_{p} / \sqrt{2}$. The surface plasmon is an oscillating sheet of charge on the metal surface. But in the direction perpendicular to the surface ( $z$ direction) the charge distribution is a simple peak, i.e., it has a monopolar character. ${ }^{1,19}$ On the contrary, there can be higher oscillation modes on the surface whose charge distribution in the $z$ direction can have a node, i.e., of dipolar or multipolar form. This is the multipole plasmon mode $\left(\omega_{m}\right)$ which exists on metal surfaces. ${ }^{1,19-25}$ Unlike the Mie plasmons for small metal particles,${ }^{26}$ the multipole surface plasmon cannot be explained by the classical Fresnel theory. The deviation from the Fresnel field is given by the $d$ parameters. ${ }^{1} d_{\perp}(\omega)$ represents the centroid of the screening density induced by a uniform field oriented normal to the surface and is given by

$$
d_{\perp}(\omega)=\frac{\int d z z n_{1}(z, \omega)}{\int d z n_{1}(z, \omega)},
$$

where $n_{1}(z, \omega)$ is the induced charge density and $z$ is the distance normal to the surface. A normal mode occurs at the pole of the surface response function which is related to $d_{\perp}(\omega) . d_{\perp}(\omega)$ exhibits a pole around $0.8 \omega_{p}$ with $\operatorname{Re} d_{\perp}(\omega)$ rapidly going through zero and $\operatorname{Im} d_{\perp}(\omega)$ exhibiting a sharp spike. ${ }^{27}$ While different physical quantities depend on $d(\omega)$, what is most important for the present purpose is that the 
total surface photoyield is proportional to $\operatorname{Im} d_{\perp}(\omega)$. Parallel to the surface, both the surface and the multipole plasmons propagate like plane waves with alternate positive and negative regions. Thus along the surface both the modes are dipolar in nature. The surface plasmon has a negative dispersion at small $q$ while the multipole plasmon has a positive dispersion. ${ }^{7}$ Also, while the multipole and the surface plasmon exist at the surface of an adlayer, the bulklike plasmon mode in an adlayer is the analog of the antisymmetric collective excitation of a finite slab and it is spatially confined to the adlayer. ${ }^{28}$

The response of a metal surface to the electromagnetic field is characterized by a rapid variation of the electric field at the metal surface when the incident frequency is between the surface $\left(\omega_{s}\right)$ and the bulk $\left(\omega_{p}\right)$ plasmon frequency. ${ }^{2,29}$ As a result, the $\nabla \cdot A$ term in the photoemission matrix element

$$
M_{i f}=\left\langle\phi_{f}|A \cdot \nabla+\nabla \cdot A| \phi_{i}\right\rangle
$$

can actually dominate the photocurrent, and lead to a large enhancement of the photoyield. In a combined theoretical and experimental study, Levinson et al. ${ }^{6}$ observed a strong enhancement of the photoyield at about $0.8 \omega_{p}$ and attributed it to the $\nabla \cdot A$ term on the basis of self-consistent field calculations within the random-phase approximation. Only much later, after Tsuei et $a l .^{7}$ had identified an additional collective excitation with an energy between $\omega_{s}$ and $\omega_{p}$ which they attributed to a multipolar mode $\omega_{m}$, did it become clear that this multipole surface plasmon is responsible for the photoemission yield enhancement for frequencies between $\omega_{s}$ and $\omega_{p}$.

The existence of a higher-order plasmon mode on metal surfaces, and its dependence on the charge-density profile was originally proposed by Bennet ${ }^{20}$ based on a hydrodynamic model calculation. In this model, the bulk charge density was assumed to decrease linearly at the surface over a distance $d$. Bennet found that for sufficiently large $d$, multipole plasmon modes appear between the surface and the bulk plasmon. Eguiluz et al. ${ }^{21}$ showed that the net charge of the multipole plasmon perpendicular to the surface integrates to zero at all points of the surface. Inglesfeld and Wikborg ${ }^{22}$ found a multipole excitation induced in a jelliumlike surface charge-density profile. Forstmann and Stenschke showed, on the basis of hydrodynamic calculations, that the consideration of longitudinal plasma waves at the metal boundary indeed leads to the increase in photoyield. ${ }^{30}$ In recent years theory, based on the random-phase approximation (RPA) or the time-dependent local-density approximation (TDLDA), has made considerable progress in the understanding of the collective excitations and in its predictive capability for simple metal surfaces and adlayers. ${ }^{1,27,31-36}$ The frequency dependence of the optical response in alkali-metal thin films has been studied by Liebsch using jellium-based TDLDA calculations. ${ }^{31}$ In these calculations, the relevant quantity that has been compared to experiment is the imaginary part of $d_{\perp}(\omega)$, which is proportional to the total photoyield, as discussed earlier. The existence of the clearly defined adlayer plasmons was related to the formation of a well-defined charge-density plateau corresponding to the alkali-metal ad- layer. For one-monolayer coverage the plasmon modes are broadened and cannot be separately observed, while for submonolayer coverage a different threshold excitation mode was postulated. ${ }^{33}$

Smooth adlayers provide a means of investigating the evolution of collective excitations with film thickness. Since the thin films exhibit the multipole surface plasmon and also carry the bulklike plasmon excitation because of the reflection barrier both at the vacuum and the thin-film-substrate interface, one can examine the relative strength of these excitations and the influence of the film and the substrate on these modes. Nearly free-electron metals like $\mathrm{Na}, \mathrm{K}$, and $\mathrm{Al}$ are ideal for such studies, since the collective electronic excitations are not affected by the lattice potential. ${ }^{15}$ The films of $\mathrm{Na}$ and $\mathrm{K}$ are particularly useful since they represent quasi-two-dimensional electron gases whose thickness can be varied over a wide range. Moreover, the alkali metals deposited on $\mathrm{Al}(111)$ at room temperature form complicated surface structures, including the recently identified $\mathrm{Al}(111)$ $(2 \times 2)$ : Na surface alloy phase..$^{37,39-42}$ Potassium forms a $\mathrm{Al}(111)-(\sqrt{3} \times \sqrt{3}) R 30^{\circ}: \mathrm{K}$ structure where the $\mathrm{K}$ atoms, depending on the deposition condition, can occupy either ontop or substitutional sites. ${ }^{41,43,44}$ These substitutional and surface alloy structures modify the shapes of the substrate $\mathrm{Al}$ charge-density profile and are expected to affect the substrate $\mathrm{Al}(111)$ multipole plasmon.

In this paper we study the photon-excited collective modes in $\mathrm{Na}$ and $\mathrm{K}$ films deposited on $\mathrm{Al}(111)$ using the method of angle- and energy-resolved photoyield (AERPY) spectroscopy. Some results for $\mathrm{Li} / \mathrm{Al}(111)$ are also discussed for comparison. The nature of the collective excitations (multipole and bulklike plasmons) in the adlayer thin films are discussed. The substrate $\mathrm{Al}(111)$ multipole excitation around $13 \mathrm{eV}$ and its dependence on adlayer growth is studied. Although there are a few studies on alkali-metal thin films, their effect on the substrate collective excitations to the best of our knowledge does not exist in the literature. We investigate the changes in frequency, full width at half maximum (FWHM), and intensity of the alkali-metal collective excitations as a function of smooth adlayer coverage. The suppression (growth) of the $\mathrm{Na}(\mathrm{K})$ plasmons by the growth of $\mathrm{K}$ layers on $\mathrm{Na} / \mathrm{Al}(111)$ is discussed to demonstrate how the susbtrate collective excitations are damped by the adlayer. In addition to the relatively simple situation of smooth adlayer growth, we study the complicated surface structures that are formed on alkali-metal deposition on $\mathrm{Al}(111)$. The motivation is to study how the collective excitations (of both adlayer and substrate) are modified due to changes in the surface charge-density profiles of these surface structures. For example, in the case of $\mathrm{K} / \mathrm{Al}(111)$ two kinds of $\mathrm{Al}(111)-(\sqrt{3} \times \sqrt{3}) R 30^{\circ}: \mathrm{K}$ phases are formed, where the $\mathrm{K}$ atoms occupy the substitutional or the on-top sites, though the symmetry remains the same. The recently identified $\mathrm{Al}(111)-(2 \times 2): \mathrm{Na}$ surface alloy phase, where the top three layers form a Na-Al-Na sandwich, has also been studied.

It should be noted that in electron-energy-loss spectroscopy (EELS) experiments, due to the presence of dominant surface plasmon excitation, the multipole plasmon signal is often difficult to observe. For example, the Al multipole plas- 
mon could not be seen by EELS, while it is clearly detected by photoyield measurements. ${ }^{6,8}$ Moreover, due to the nonanalytic dispersion of the plasmon modes near $q=0$, EELS is not an appropriate technique to study the plasmon modes in the long-wavelength limit. ${ }^{14}$ Since the photon carries almost zero momentum, photoyield based techniques are best suited in this regime. The additional advantage is that, unlike EELS, the dominant surface plasmon is not excited by the photons and the weaker surface modes like the multipole plasmon can be clearly observed.

The outline of the paper is as follows. In Sec. II the experimental procedure is described. The results and discussion section (Sec. III) is divided into three subsections which describe (i) the collective excitations in smooth alkali-metal adlayers, (ii) the influence of smooth adlayer growth on substrate collective excitations, and (iii) the influence of adatom induced surface structures on the adlayer and substrate collective excitations. The conclusion is given in Sec.IV.

\section{EXPERIMENT}

The measurements were performed at the BerlinerElektrononen- Speicherring- Gesellschaft- für- Synchrotronstrahlung (BESSY-I) with the 1-m Seya Namioka monochromator equipped with two spherical gratings. The experiments were performed using a commercial angle-resolved electron spectrometer (ADES400 from VG, U.K.) at a base pressure of $6 \times 10^{-11}$ mbar. An electropolished $\mathrm{Al}(111)$ crystal was cleaned by repeated cycles of sputtering using $0.5-1-\mathrm{KeV}$ $\mathrm{Ne}$ atoms and subsequent annealing to regenerate surface order. The surface order was monitored using low-energy electron diffraction (LEED) and a sharp (1×1) LEED pattern was observed for the clean surface.

The adlayers were prepared by depositing the alkali metals over the clean $\mathrm{Al}(111)$ substrate at $100 \mathrm{~K}$ (for smooth adlayer growth) and at room temperature (for surface alloy related phases). Well-degassed alkali-metal dispensers (SAES getters) were ohmically heated and the pressure inside the chamber remained below $3 \times 10^{-10}$ mbar during deposition. Coverage was calibrated on the basis of existing data on the variation of the work function, ${ }^{42}$ core level line shape in $\mathrm{K}$ layers and for thicker layers by deposition time. A layer by layer growth was established and the surface roughness was negligible since the surface plasmon was not observed. We define one monolayer (ML) in terms of a fully occupied (110) plane of the alkali-metal bulk crystal. Then, in terms of the number of atoms in the $\operatorname{Al}(111)$ plane $(\Theta=1)$ the corresponding coverages for one full layer are: $\Theta=0.45$ for $\mathrm{K}$ and $\Theta=0.53$ for Na. For deposition at $100-\mathrm{K}$ substrate temperature [low-temperature (LT) deposition], the $\mathrm{Na}$ / $\operatorname{Al}(111)$ system initially exhibits a LEED pattern with a $\frac{4}{3} \times$ $\frac{4}{3}$ symmetry. At higher coverages the growth is epitaxial with a low background hexagonal $1 \times 1$ pattern. For $\mathrm{K} / \mathrm{Al}(111)$, a $(\sqrt{3} \times \sqrt{3}) R 30^{\circ}$ structure is initially formed with the $\mathrm{K}$ atoms occupying the on top sites, which turns to a nonepitaxial growth for high coverages. The on-top $\operatorname{Al}(111)-(\sqrt{3}$ $\times \sqrt{3}) R 30^{\circ}: \mathrm{K}$ structure was obtained by deposition of $\mathrm{K}$ at $100 \mathrm{~K}$, and the substitutional $\operatorname{Al}(111)-(\sqrt{3} \times \sqrt{3}) R 30^{\circ}: \mathrm{K}$ structure was formed by subsequently heating up to room temperature. For the room-temperature deposition of $\mathrm{Na}$, the $\mathrm{Al}(111)-(\sqrt{3} \times \sqrt{3}) R 30^{\circ}: \mathrm{Na}$ structure is initially formed at $\Theta=0.33$, and on futher deposition the $\mathrm{Al}(111)-(2 \times 2)$ : Na surface alloy phase is formed at $\Theta=0.5$. Interestingly, even after reaching the $(2 \times 2)$ phase further deposition of $\mathrm{Na}$ was possible which resulted in very faint $(2 \times 2)$ spots, beyond which no further $\mathrm{Na}$ could be deposited.

We have performed the experiments using the angle- and energy resolved photoyield (AERPY) technique ${ }^{6,8}$ by recording intensity at binding energies near $E_{F}$ as a function of $h \nu$ in the constant initial state (CIS) mode in the normal emission geometry. The energy resolution was $0.1-0.2 \mathrm{eV}$ in the studied energy range and the angular resolution was $3^{\circ}$. The advantage of AERPY (where the photoyield from the Fermi level is measured at normal emission) over total yield measurement is that it does not have a contribution from inelastically scattered secondary electrons, which is difficult to analyze and may also depend on surface quality and sample preparation history. The $p$-polarized photons from the synchrotron ring were incident on the sample at $45^{\circ}$ to the surface normal. Energy distribution curves (EDC's) in normal emission as a function of photon energy were also recorded, and the intensity at $E_{F}$ was plotted as a function of photon energy to give the AERPY spectra. However, since this method is more time consuming and the alkali-metal layers are highly prone to contamination, the former approach of recording AERPY spectra in the CIS mode have been followed. The AERPY data were collected at binding energies $0.1 \mathrm{eV}(\mathrm{K})$ and $0.3 \mathrm{eV}(\mathrm{Na})$ below $E_{F}$.

The spectra have been normalized with respect to the incident photon flux which shows characteristic variation for the two different gratings. The photon flux was monitored simultaneously with the CIS spectrum by recording the photocurrent induced in a $\mathrm{Au}$ mesh with $95 \%$ transmittance in the path of the photon beam. However, since the gold yield goes to zero below its work function, the photon flux was also recorded using a GaAsP diode which is sensitive down to about $2 \mathrm{eV}$. The AERPY spectra were corrected for the variation of the analyzer transmission function with electron kinetic energy as well as for higher-order light. Even though the spectra are not normalized in absolute terms, they can be compared to each other on the same intensity scale.

\section{RESULTS AND DISCUSSION}

\section{A. Collective excitations in smooth alkali-metal adlayers}

$\mathrm{Na}$ related collective excitations are clearly observed on the $\mathrm{Al}(111)$ substrate and they show an interesting dependence on the adlayer thickness (Fig. 1). The substrate $\mathrm{Al}(111)$ spectrum is essentially flat, with a weakly rising intensity towards the work-function cutoff at $4.8 \mathrm{eV}$. With deposition of $0.5-\mathrm{ML} \mathrm{Na}$ at low temperature $(100 \mathrm{~K})$, there is an initial decrease in the work function $(\Delta \phi=1.5 \mathrm{eV})$ resulting in a shift of the cutoff to $3.3 \mathrm{eV}$. This shift is consistent with the reduction of the work function induced by the adsorption of $\mathrm{Na}$ on $\mathrm{Al},{ }^{42}$ which reaches a minimum at $\approx 0.5 \mathrm{ML}$. The difference between the clean and 0.5-ML spectra is a gradual increase of intensity in the latter as the photon energy decreases from 6 to $3.3 \mathrm{eV}$. At 1.0-ML coverage, a weak feature 


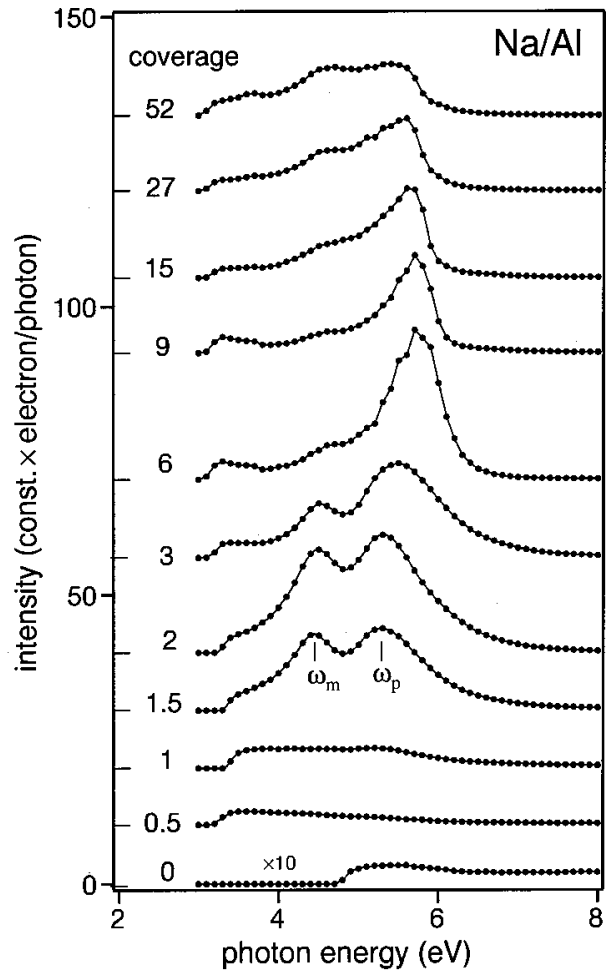

FIG. 1. Angle- and energy resolved photoyield (AERPY) spectra for $\mathrm{Na} / \mathrm{Al}(111)$ as function of adlayer coverage. The spectra are shifted with respect to each other and the zero of each spectrum is indicated on the left vertical axis. $\omega_{m}$ and $\omega_{p}$ are the $\mathrm{Na}$ adlayer related multipole surface plasmon and bulklike plasmon excitations, respectively.

around $5.3 \mathrm{eV}$ is observed. As the second layer starts to build up, this feature shows a dramatic increase in intensity and develops into a well defined peak at $5.3 \mathrm{eV}$ while another peak appears at $4.5 \mathrm{eV}$. We attribute the peak at $4.5 \mathrm{eV}$ to be due to the multipole plasmon excitations at the adlayervacuum interface, while the $5.3-\mathrm{eV}$ peak is due to a bulklike plasmon mode in the adlayer. ${ }^{33}$ With increasing coverage, the ratio of the bulk to multipole plasmon intensity decreases and this trend continues up to the highest coverage that we have studied.

The growth of the K collective excitations as function of layer thickness is shown in Fig. 2. For one-monolayer coverage, since the ground-state charge density is not diffuse enough the adlayer bulk and multipole plasmon modes are heavily broadened, and a mixed plasmon peak appears around $3 \mathrm{eV}$, as shown by previous TDLDA calculations. ${ }^{33}$ Beyond $1 \mathrm{ML}$ a shoulder occurs at $3.5 \mathrm{eV}$, which at $2 \mathrm{ML}$ 's develops into two well defined peaks at 3.2 and $3.6 \mathrm{eV}$, corresponding to the multipole and the bulklike plasmon modes, respectively. ${ }^{15}$ The work-function cutoff for $\mathrm{K}$ layers is smaller than the lowest energy that can be reached by the monochromator and hence not seen in the spectra. With increasing coverage the bulk plasmon mode clearly decreases in intensity, and for the highest coverage ( $40 \mathrm{ML}$ 's) it is almost fully suppressed and a weak feature above $\omega_{p}$ at $4 \mathrm{eV}$ is observed. A feature around a similar relative frequency $\left(1.05 \omega_{p}\right)$ has been observed for $\mathrm{Al}(111)$ (the weak feature at

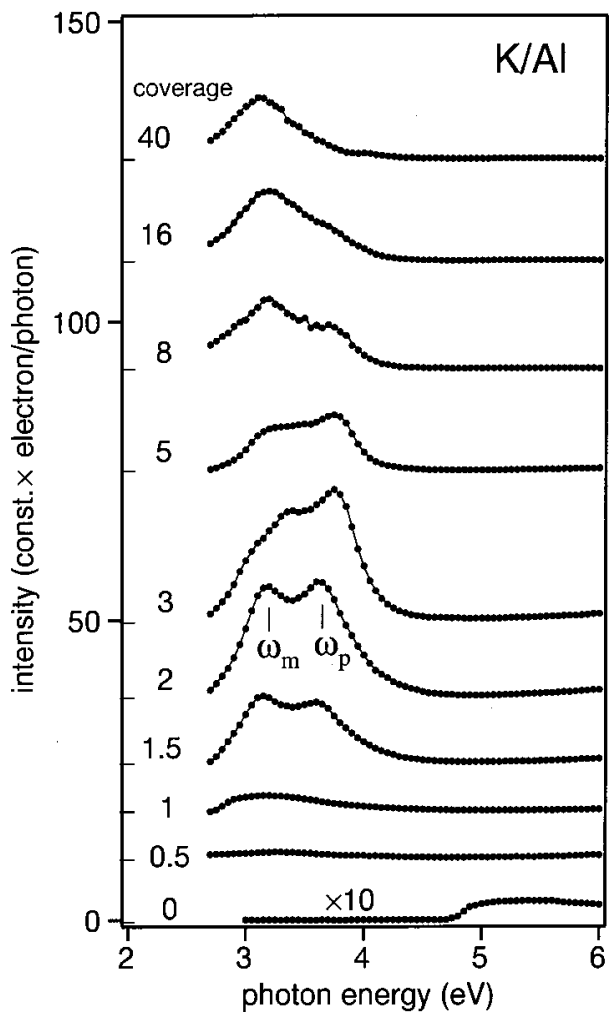

FIG. 2. AERPY spectra for $\mathrm{K} / \mathrm{Al}(111)$ as function of adlayer coverage. The spectra are shifted with respect to each other and the zero of each spectrum is indicated on the left vertical axis. $\omega_{m}$ and $\omega_{p}$ are the K adlayer related multipole surface plasmon and bulklike plasmon excitations, respectively.

$16 \mathrm{eV}$ in Fig. 6) and has been attributed to excitation of longitudinal bulk plasmons $\left(\omega \geqslant \omega_{p}\right)$ with $q \geqslant 0 .{ }^{8}$

We compare in Fig. 3 the Na/Al AERPY data for 0.5-, 1-, and 2-ML coverages with the results of TDLDA calculations. ${ }^{31,33}$ The calculations have been performed both for jellium adlayers and realistic alkali-metal overlayers on semi-infinite jellium representing the $\mathrm{Al}$ substrate. In the realistic calculation, the alkali-metal cores are represented by norm-conserving pseudopotentials and the ground state is calculated within the local-density approximation of the density-functional theory (DFT). The dynamical response to the incident electric field is calculated within TDLDA. The total photoyield is calculated by evaluating $\operatorname{Im} d_{\perp}(\omega)$ and this is compared with the experimental AERPY data. For an adsorbate double layer, the observation of the bulk and multipole plasmon modes provides an experimental verification of the theory. In order to compare the line shape, the theoretical spectra for 2-ML coverage are shifted to lower energy by $0.5 \mathrm{eV}$ to match the experimental bulk-plasmon peak at $5.3 \mathrm{eV}$. The line shape as well as the relative intensity are in good agreement with theory. However, the experimental FWHM of 0.5 and $1.2 \mathrm{eV}$ for multipole and bulk plasmons, respectively, for 2-ML coverage are slightly underestimated by TDLDA ( 0.4 and $0.8 \mathrm{eV}$, respectively). ${ }^{33}$ This is probably due to mechanisms such as electron scattering due to defects and steps on a real surface, or damping due to interband transitions which are not considered in the theory. The over- 


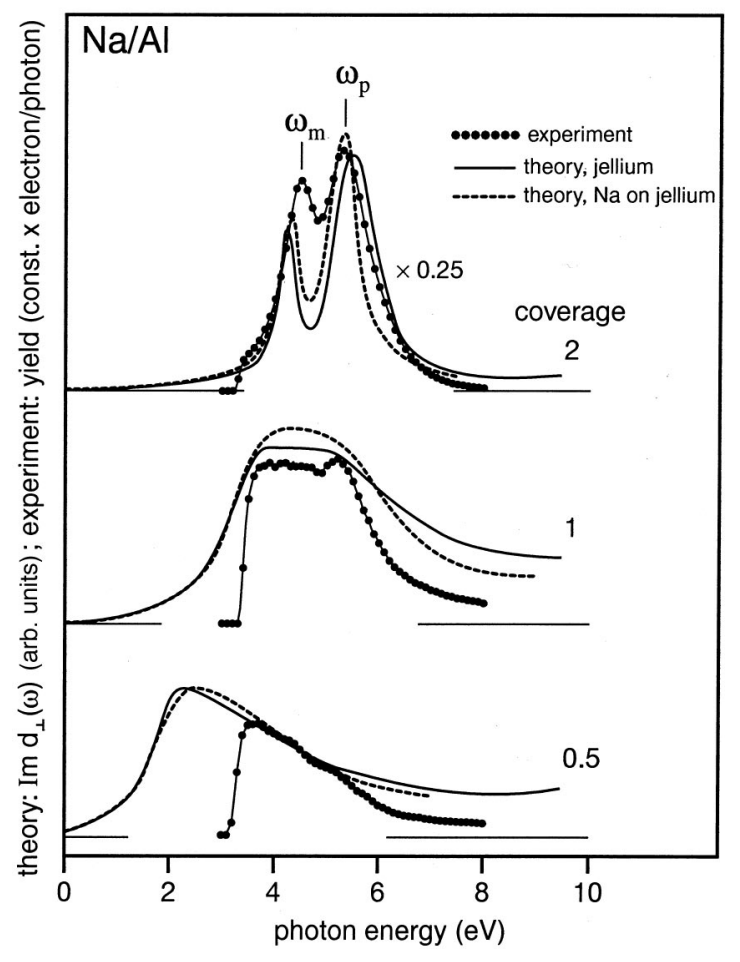

FIG. 3. Comparison of the Na/Al experimental AERPY spectra with theoretical total photoabsorption calculations (see text) based on TDLDA from Ref. 33 for three coverages (0.5, 1, and 2 ML's).

estimation of the plasmon frequency by $0.5 \mathrm{eV}$ is probably due to the polarization of the core electrons which tend to screen the plasmon oscillations. ${ }^{45,46}$ For 1-ML coverage, there is no well defined charge-density plateau corresponding to the adlayer and the substrate-adsorbate and the adsorbatevacuum interfaces are not clearly defined. Hence the bulk and the multipole plasmon modes become heavily broadened due to the coupling with electron-hole pairs, giving rise to a broad peak in the threshold region. ${ }^{33}$ The experiment exhibits a rising intensity towards the work-function cutoff corresponding to this mixed plasmon mode. The weak feature at $5.3 \mathrm{eV}$ is related to the onset of the bulk-plasmon peak, possibly due to double layer formation in some parts of the surface. In the submonolayer region (for example 0.5 ML in Fig. 3), an increasing intensity towards the work-function cutoff is observed in the experiment. $\operatorname{Im} d_{\perp}(\omega)$ also shows a similar behavior. This enhancement has been assigned by Ishida and Liebsch to a different type of collective excitation called the threshold excitation. ${ }^{33}$ These authors showed that the threshold excitation is a consequence of the screening of the total effective potential $\left(\Phi_{s c f}\right)$ on the substrate side of the adlayer and a higher cross section of electron-hole excitations at around $h \nu=\Phi$. In fact, it is interesting to note that if $\Phi_{s c f}$ is replaced by the unscreened external potential in the expression for $\operatorname{Im} d_{\perp}(\omega)$, the excitation spectra at all the coverages for which calculations have been done are smooth curves without any features. Thus the bulk and the multipole plasmon as well as the threshold excitation mode arise due to the dynamical screening of the external electric field in the adlayer.
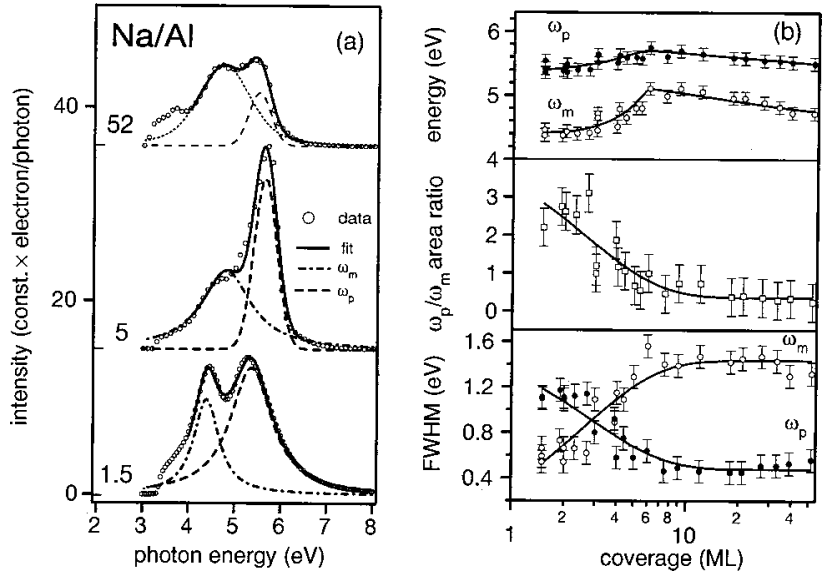

FIG. 4. (a) A least-squares fit to the experimental Na/Al AERPY spectra (open circles) for 1.5-, 5-, and 52-ML coverage. The thick line corresponds to the total fitted spectrum, while the dot-dashed and the dashed lines show the multipole and the bulk-plasmon components, respectively. (b) The variation of the plasmon frequencies (upper panel), the bulk to multipole plasmon area ratio (middle panel, open squares), and the FWHM (lower panel) of the Na multipole (open circle), and bulk plasmon (filled circle) are shown as function of coverage. Solid lines serve as a guide to the eye.

In order to find whether the relative intensity, position, and width of the adlayer plasmons exhibit a systematic variation with coverage, we have simulated the experimental spectra in Fig. 1 with two Voigt functions (corresponding to the bulk and multipole plasmons) and a flat background, using a least-square minimization routine. The choice of the Voigt function line shape gave better fits than either a purely Lorenzian or a Gaussian function. Both the Lorenzian as well as the Gaussian component of the Voigt function linewidth were varied during the fitting. The least-square minimization is done over a restricted energy range $(4-6.5 \mathrm{eV})$ where the plasmon intensities mostly contribute. For example, below $4 \mathrm{eV}$ (which is roughly the inflection point) the threshold excitation can alter the line shape. No theoretical results exist on the line shape variation of the photon excited adlayer plasmons.

Representative fits for three different coverages $(1.5,5$, and 52 ML's) with the bulk- and multipole plasmon components are shown in Fig. 4(a). The bulklike plasmon is dominant at the onset (1.5 ML's), but its width and intensity decrease at higher coverages. The width of the multipole plasmon, on the other hand, increases with coverage. The variation of the bulk- and multipole plasmon energy, the ratio of the bulk to multipole plasmon peak area, and the full width at half maximum (FWHM) as a function of coverage are shown in Fig. 4(b). The bulk-plasmon energy exhibits a blue shift from 5.3 to $5.7 \mathrm{eV}$ up to a coverage of about 5 ML's, beyond which a gradual redshift to $\sim 5.5 \mathrm{eV}$ is observed. It should be noted that this value is close to $\omega_{p}$ for bulk $\mathrm{Na}$ from previous optical studies (see, for example, Table V of Ref. 12). This shift is larger than the error bar $( \pm 0.1 \mathrm{eV})$ which has been estimated from the scatter in the peak position for the same coverage in different measurements. The multipole plasmon also exhibits a blue shift 

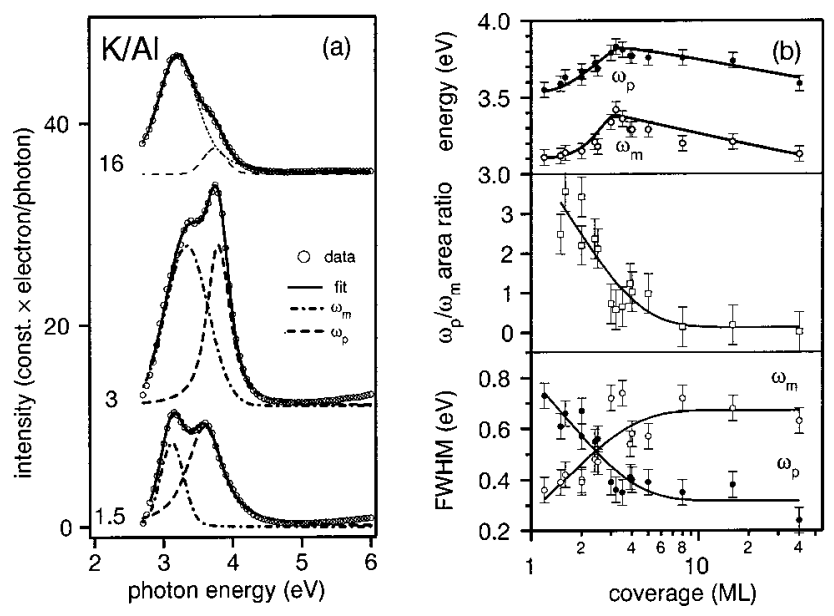

FIG. 5. (a) A least-squares fit to the experimental K/Al AERPY spectra (open circles) for 1.5-, 3-, and 16-ML coverage. The thick line corresponds to the total fitted spectrum, while the dot-dashed and the dashed lines show the multipole and the bulk-plasmon components, respectively. (b) The variation of the plasmon frequencies (upper panel), the bulk to multipole plasmon area ratio (middle panel, open squares), and the FWHM (lower panel) of the K multipole (open circle) and bulk plasmon (filled circle) are shown as function of coverage. Solid lines serve as a guide to the eye.

(from 4.4 to $5 \mathrm{eV}$ ) for lower coverages and a subsequent redshift to $4.7 \mathrm{eV}$ for thicker coverage. A close look at the experimental data in Fig. 1 also shows this characteristic shift of the plasmon energies with coverage.

The ratio of the total areas under the bulk and multipole plasmon peaks and the variation of their FWHM are shown in the middle and lower panel of Fig. 4(b), respectively. Although the bulk plasmon is most intense at the onset (1.5-2 ML's), its width (i.e., FWHM) and intensity (i.e., area under the peak) decrease with coverage, whereas the multipole plasmon width and intensity increase. Thus a steep decrease of the intensity ratio is observed up to about 5 ML's, beyond which the decrease is gradual. For the highest coverage, the multipole plasmon is more than four times as intense than the bulk plasmon. There is an initial increase in the multipole plasmon width from 0.5 to $1.4 \mathrm{eV}$, while the bulk-plasmon width decreases from about 1.2 to $0.5 \mathrm{eV}$ in the range of 1.5-5-ML coverage. A comparison of the spectra and the component bulk- and multipole plasmon line shapes [Fig. 4(a)] between 1.5 and 5 ML's shows that the higher energy tail of the bulk plasmon in the former extends to almost about $8 \mathrm{eV}$ in contrast to the latter. This indicates that the bulk plasmon is indeed narrower in the 5-ML spectrum, as shown by the results of the fitting exercise. Beyond about 5 ML's, the multipole and bulk-plasmon FWHM's do not vary significantly, their values being 1.4 and $0.5 \mathrm{eV}$, respectively.

The fit to the AERPY spectra in K/Al(111) for 1.5-, 3-, and 16-ML coverage are shown in Fig. 5(a). As in the $\mathrm{Na}$ layers, the spectra are fitted with two Voigt functions, corresponding to the multipole and the bulk plasmon, using a least-square fitting procedure over the energy range of 2.8$4.3 \mathrm{eV}$. The variation of the plasmon frequencies, the bulk to multipole plasmon intensity ratio and the plasmon FWHM's as a function of coverage are shown in Fig. 5(b). The shift of the $\mathrm{K}$ plasmon frequencies is quite similar to that of the $\mathrm{Na}$ layers. Both $\mathrm{K}$ bulk and multipole plasmons exhibit a blue shift up to about 5 ML's beyond which a gradual redshift is observed. The bulk plasmon shifts from 3.55 to $3.8 \mathrm{eV}$ for the coverage range of 1.2-4 ML's and finally to $3.6 \mathrm{eV}$ for the thickest coverage (40 ML's). The multipole plasmon follows the bulk plasmon with an initial blue shift from 3.1 to $3.4 \mathrm{eV}$ and then a redshift back to $3.1 \mathrm{eV}$ for the thick layers. For a thick K layer, the frequency of the multipole plasmon from EELS measurements is $3.2 \mathrm{eV}$ at $q_{\|}=0.03$. $^{7}$ The $\mathrm{K}$ multipole and bulk-plasmon frequencies $(3.15$ and $3.65 \mathrm{eV}$, respectively) at 2-ML coverage are in agreement with recent total photoyield measurements at the same coverage. ${ }^{14}$

The ratio of the $\mathrm{K}$ bulk to multipole plasmon intensity [middle panel in Fig. 5(b)] decreases steeply up to about 5-6 ML's and shows a subsequent gradual decrease up to the highest coverage where it is nearly zero because of the almost complete attenuation of the bulk plasmon. The FWHM of the multipole plasmon [lower panel in Fig. 5(b)] shows an initial increase from about 0.35 to $0.65 \mathrm{eV}$, whereafter at higher coverages it does not further change. The bulkplasmon FWHM, on the other hand, decreases from 0.7 to $0.3 \mathrm{eV}$ over the studied coverage range. The relative changes in the intensity and width of the plasmons can be seen in Fig. 5(a). For example, between the 1.5- and 3-ML spectra, the multipole plasmon clearly increases in width. Although the bulk-plasmon peak height is higher in the 3-ML spectrum, the multipole plasmon has a larger weight due to an increased FWHM. For the 40-ML spectrum, the bulklike plasmon is almost fully suppressed; however, the multipole plasmon has an asymmetric higher energy tail which is probably due to the remnant adlayer bulklike plasmon appearing at 3.6 $\mathrm{eV}$, as shown from the fitting.

The collective excitations for both $\mathrm{Na}$ and $\mathrm{K}$ adlayers thus behave similarly. In both cases, with increasing thickness there is an initial blue shift of the plasmon frequency up to about 5 ML's beyond which a redshift is observed. The origin of the redshift can be related to the dispersion of the bulk plasmon with wave vector $q$. The bulk plasmon mode is the analog of the antisymmetric collective excitation of a finite slab. ${ }^{28}$ A recent calculation ${ }^{15,36}$ of the induced charge density for two and three layers of $\mathrm{Li}$ on $\mathrm{Al}$ at $h \nu=\omega_{p}$ shows that it oscillates with a wavelength somewhat larger than the interlayer distance. Similar results have been obtained for two layers of $\mathrm{K}$ on $\mathrm{Al}^{14}$ These results show that the bulkplasmon mode corresponds to a standing wave extending across the entire layer. For suspended thin films, bulkplasmon resonances have been predicted for $\omega \geqslant \omega_{p}$ with $\lambda_{\perp}=2 a / n$, where $\lambda_{\perp}$ is the wavelength of the plasmon perpendicular to the surface, $n$ is an odd positive number, and $a$ is the thickness of the layer. ${ }^{47}$ Such higher-order modes were experimentally detected as weak features in $\mathrm{K}$ films grown on quartz by differentiating the total yield with photon energy. ${ }^{48}$ These resonances follow a quadratic dispersion given by

$$
\omega_{p}(q)=\omega_{p}+\alpha q^{2}
$$

where $\alpha=0.6 \epsilon_{F} / \omega_{p} \cdot{ }^{1}$ The bulk-plasmon resonance that we observe at $\omega_{p}$ should also exhibit a similar dispersion with $q$, 
i.e., as $q$ decreases the frequency should decrease. Thus, with increasing thickness of the adlayer, the wavelength of the plasmon mode increases, resulting in the redshift of the bulkplasmon frequency. Moreover, in agreement with the present experimental data, a hydrodynamic calculation for the metalllic adlayer/substrate system showed a redshift in the frequency of the longitudinal plasmon modes in the adlayer with increasing thickness. ${ }^{49}$ It should be noted that we do not observe the higher-order modes above $\omega_{p}$, nor are they predicted by the TDLDA calculations ${ }^{33}$ for the alkali metal/Al system probably because of the larger damping of the plasmon modes at the alkali-metal-Al interface.

The reason for the observed blue shift in the lower coverage region is probably related to the smooth density profiles at the adsorbate-vacuum and the adsorbate-substrate interfaces which would lower the true bulk-plasmon frequency. ${ }^{1}$ This effect will be more pronounced at lower coverages and decreases with the increasing thickness of the adlayer. In fact, the experimentally observed negative dispersion of the surface plasmon at small $q(\leqslant 0.15 \AA)$ has been shown to be related to the smooth surface density profile. ${ }^{35}$ The blue shift of the bulklike plasmon observed here is due to its lower frequency at onset coverage (2 ML's) and a subsequent increase in frequency as the layer thickness increases and the adsorbate interface effect becomes negligible.

Although the multipole plasmon exists only at the surface, it exhibits a similar dispersion with layer thickness as the bulk plasmon, and this shows that these two modes in the adlayer are coupled. In fact, from a large body of available literature, in the retarded $q \sim 0$ limit, the multipole plasmon has been found to occur at $\beta \omega_{p}$ independent of the charge density, with $\beta$ roughly around 0.8 . The physical reason for the dependence of $\omega_{m}$ on $\omega_{p}$, which is independent of the electron density, is not fully understood. ${ }^{1}$ However, the value of $\beta$ is of importance from a theoretical point of view since calculations based on the random-phase approximation (RPA) give a higher value of $\beta$ compared to TDLDA. For K, for example, TDLDA predicts $\beta$ to be 0.82 , while RPA gives a value of $0.89 .{ }^{27}$ We find for the K layers $0.86 \leqslant \beta \leqslant 0.89$, and for the highest coverage $\beta=0.87$ which is closer to the value predicted by RPA. For $\mathrm{Na}$ layers, we find $0.8 \leqslant \beta \leqslant 0.89$, the value at the highest coverage being 0.86 . In the case of clean $\mathrm{Al}$, the multipole plasmon occurs around $13 \mathrm{eV}$, which corresponds to a $\beta$ value of 0.85 . Thus, for the nearly free-electron metals, our results indicate that on an average the multipole plasmon occurs around $0.85 \omega_{p}$ which is somewhat higher than the generally accepted value of $0.8 \omega_{p}$. In real metals, $\omega_{p}$ is probably reduced by the core polarization effect, which causes an overall lowering of the bulklike plasmon frequency. ${ }^{1}$ The multipole plasmon, on the other hand, being located at the surface, is less affected by the core polarization effect and its frequency is not lowered with respect to $\omega_{p}$ leading to an increase in $\beta$.

The FWHM of the adlayer bulklike plasmon decreases with increasing coverage [Figs. 4(b) and 5(b)]. While no quantitative calculations exist, a plausible reason for this behavior could be that the bulk plasmon couples less with the excitations at the adlayer-substrate and the adlayer-vacuum interface as the thickness increases. Thus the possible decay
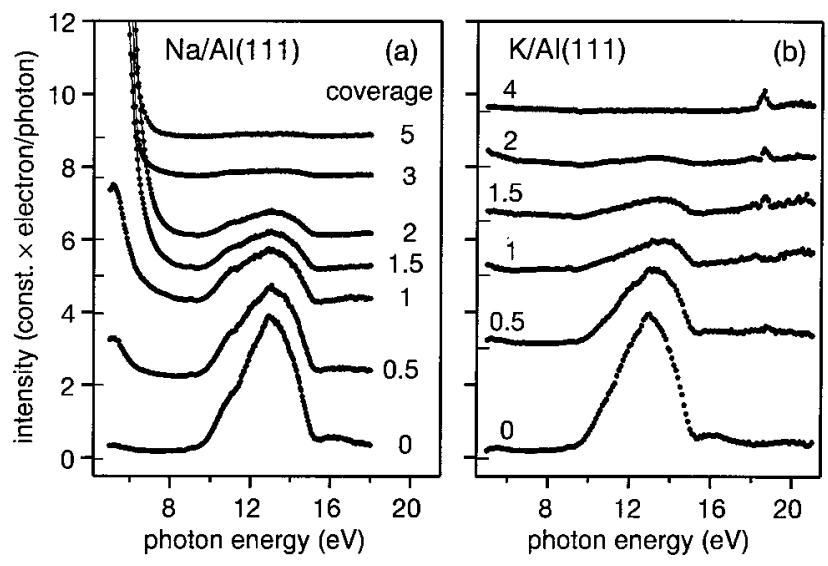

FIG. 6. AERPY spectra showing the substrate $\mathrm{Al}(111)$ multipole plasmon attenuation as function of (a) $\mathrm{Na}$ and (b) $\mathrm{K}$ coverage. The spectra are shifted with respect to each other, and the zero levels are indicated on the left vertical axis.

channels are reduced, resulting in its narrowing. The multipole plasmon, being located at the surface, will decay via excitations at the adlayer-vacuum interface which are evidently enhanced since its width increases with coverage.

The physical origin of the adlayer bulklike plasmon is the coupling of the transverse electromagnetic field and the longitudinal fields at the adlayer substrate region, and should occur quite generally at interfaces between media. ${ }^{50}$ The bulk-plasmon standing waves in the adlayer are set up due to reflection of the plasmon wave at the substrate-adsorbate and the adsorbate-vacuum interface. With increasing adlayer thickness, the electrostatic coupling between these two interfaces decreases, leading to reduction of the bulklike plasmon intensity. We indeed find from Figs. 4(b) and 5(b) that the ratio of the bulk to multipole plasmon intensity decreases with coverage for both $\mathrm{Na}$ and $\mathrm{K}$ adlayers. For semi-infinite surfaces, it has been theoretically shown that the surface photoabsorption is given by ${ }^{2}$

$$
Y=\left(1-\omega_{p}^{2} / \omega^{2}\right) \operatorname{Im} d_{\perp}(\omega),
$$

where the prefactor is related to the Fresnel field. Since the surface photoyield vanishes at $\omega_{p}$, the decrease in the adlayer bulklike plasmon intensity with coverage is consistent with the theoretical expectation. In the case of $\mathrm{K}$, at $40 \mathrm{ML}$ 's the adlayer bulk plasmon (at $3.6 \mathrm{eV}$ ) is almost fully suppressed, and a weak minimum at $3.9 \mathrm{eV}$ is observed (see Fig. 2). This dip can be regarded as the signature of the vanishing photoyield at the bulk-plasmon frequency for the semiinfinite solid as also seen for clean $\mathrm{Al}$ (see Fig. 6).

\section{B. Influence of smooth adlayer growth on substrate collective excitations}

We now turn to the question of what happens to the substrate collective excitation as a function of adlayer thickness. The AERPY spectrum for a clean $\mathrm{Al}(111)$ substrate exhibits a peak at $13 \mathrm{eV}$ (Fig. 6), which is related to the multipole plasmon excitation at the surface. At the bulk-plasmon frequency $(\sim 15 \mathrm{eV})$, a minimum occurs because of the vanishing surface photoabsorption, as discussed earlier. The weak 


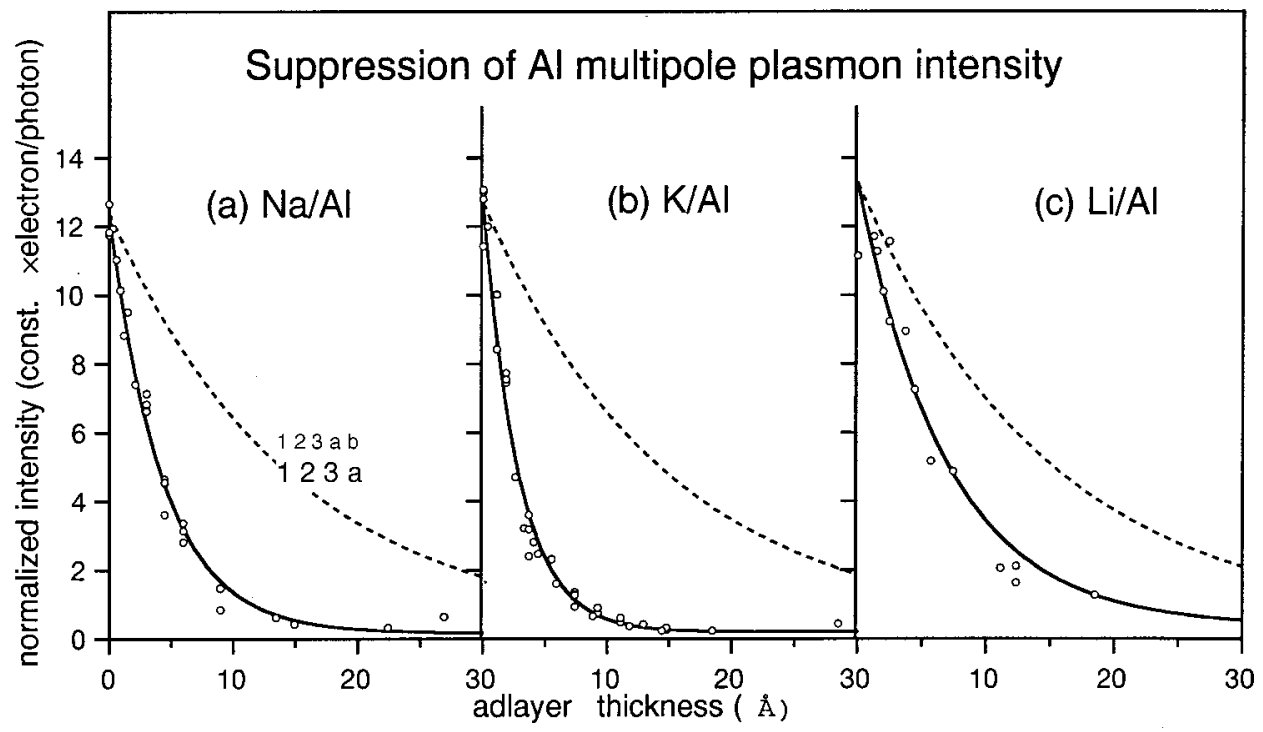

FIG. 7. Area under the $\mathrm{Al}(111)$ multipole plasmon peak, plotted as a function of (a) $\mathrm{Na}$, (b) $\mathrm{K}$, and (c) Li adlayer coverage. The solid line is an exponential fit to the data, while the dashed line shows the photoelectron inelastic scattering related attenuation curve, assuming a mean free path of $15 \AA$. feature above $\omega_{p}$ at $16 \mathrm{eV}$ is related to the excitation of the longitudinal plasmon with $q \geqslant 0$ as discussed elsewhere. ${ }^{8}$

We show in Fig. 6 a series of AERPY spectra for (a) $\mathrm{Na} / \mathrm{Al}(111)$ and (b) $\mathrm{K} / \mathrm{Al}(111)$ for the photon energy range corresponding to the Al multipole plasmon. For both systems, we find that the $\mathrm{Al}$ multipole plasmon is suppressed in intensity with increasing alkali-metal coverage, and by 4-5 ML's is almost fully attenuated. Although the Al multipole intensity decreases with adlayer growth, its position remains unchanged and there is hardly any change in the line shape. The feature around $18.5 \mathrm{eV}$ in Fig. 6(b) arises from the $\mathrm{K} 3 p$ related $M_{2,3^{-}} V V$ Auger transition moving through $E_{F}$ as the photon energy is changed. No other collective excitations corresponding to charge densities between $\mathrm{Al}\left(r_{s}=2.0\right)$ and $\mathrm{Na}\left(r_{s}=3.93\right)$ or $\mathrm{K}\left(r_{s}=4.86\right)$ appear in the spectra. The rising intensity in Fig. 6(a) around $6 \mathrm{eV}$ is due to the Na plasmons.

For a coverage of up to two adlayers, the substrate Al multipole plasmon, although attenuated, still exhibits substantial intensity. This is surprising because it is generally accepted $^{1}$ that the multipole plasmon excitation occurs at the substrate-vacuum interface, which in this case no longer exists due to alkali-metal adlayers. To understand this effect, we note that in the photon energy region where the Al multipole plasmon is excited, the $\mathrm{Na}(\mathrm{K})$ layers are fully transparent. Thus, even in the presence of the alkali metal layer, the $\mathrm{Al}$ charge density tries to screen the incident electromagnetic field as in the clean metal, and in this process sets up the multipole plasmon oscillation. The existence of the Al multipole plasmon up to about 2 ML's of alkali-metal growth is significant because it shows that the multipole plasmon can also exist as an interface plasmon, although diminished in intensity. The present observation is supported by a jellium-based calculation by Liebsch ${ }^{31}$ for monolayers of $\mathrm{Na}$, $\mathrm{K}$, and $\mathrm{Cs}$ on $\mathrm{Al}$ which showed the simultaneous existence of both the alkali metal as well as the Al related collective excitations. Compared to Al, one monolayer of alkali metals on $\mathrm{Al}$ was shown to attenuate the $\mathrm{Al}$ multipole plasmon without any shift in its frequency. ${ }^{31}$
For adlayer coverages $>2$ ML's, the substrate Al multipole plasmon is almost fully suppressed due to the formation of the well defined charge-density plateau corresponding to the adlayer, which can sustain the fully grown adlayerrelated collective excitations. In Fig. 7 the area under the $\mathrm{Al}$ multipole plasmon peak as function of alkali metal coverage for (a) $\mathrm{Na}$, (b) $\mathrm{K}$, and (c) Li adlayers is plotted. We have fitted the Al multipole plasmon intensity (I) using the following exponential function

$$
I=k_{0}+k_{1} \times \exp ^{-\left(k_{2} \times z\right)},
$$

where $z$ is the thickness of the layer in Angstroms and $k_{0}$, $k_{1}$, and $k_{2}$ are adjustable parameters. The thickness of the $\mathrm{K}$, $\mathrm{Na}$, and $\mathrm{Li}$ adlayers are taken to be $3.7,2.99$, and $2.47 \AA$, respectively, corresponding to the closed packed growth. Larger value of $k_{2}$ would imply a stronger damping of the $\mathrm{Al}$ multipole plasmon. From the fitting, we obtain the values of $k_{2}$ to be $0.35,0.23$, and 0.14 for $\mathrm{K}, \mathrm{Na}$, and $\mathrm{Li}$, respectively. This implies that for the same adlayer thickness, the rate of suppression of the Al multipole plasmon systematically increases from Li to $\mathrm{K}$. The explanation of this effect is probably related to the mechanism of the substrate multipole plasmon damping by the adlayer and further theoretical studies are required to explain this.

The damping of the signal from the substrate is partly caused by the inelastic scattering of the photoemitted electrons from the substrate by the adlayer. This is related to the technique of measurement and can attenuate the Al multipole plasmon intensity. The intensity of the electrons emitted from near the Fermi level is measured in the experiment, which if inelastically scattered would occur as the secondary background at higher binding energies. However, the cross section of the inelastic scattering is small in these low photon energies $(13 \mathrm{eV})$ which is manifested by a large mean free path $\left(\lambda_{m f p}\right)$ of about $15 \AA{ }^{51,52}$ Since the inelastic scattering also leads to an exponential decay, its effect is shown (dashed curves in Fig. 7) by taking $k_{2}=1 / \lambda_{m f p}=.067$ in the above expression used for fitting, keeping the other parameters the same. As is evident from Fig. 7, the attenuation rate 


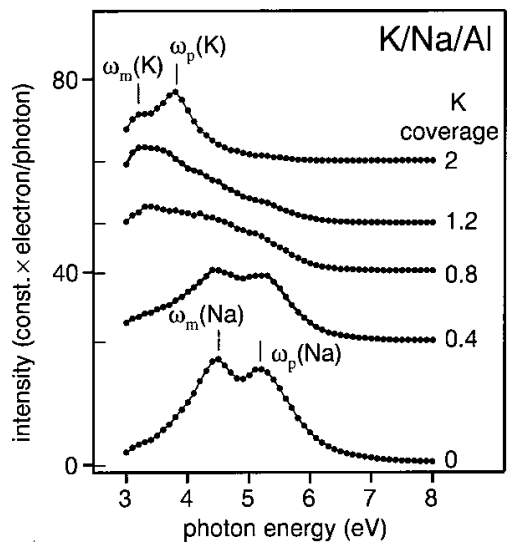

FIG. 8. Evolution of the AERPY spectra with deposition of $\mathrm{K}$ on 1.5-ML Na adlayer on $\mathrm{Al}(111)$. The Na related multipole and bulk plasmon peaks $\left[\omega_{m}(\mathrm{Na})\right.$ and $\left.\omega_{p}(\mathrm{Na})\right]$ are almost fully suppressed by 2 -ML $\mathrm{K}$, and the $\mathrm{K}$ related collective excitations $\left[\omega_{m}(\mathrm{~K})\right.$ and $\left.\omega_{p}(\mathrm{~K})\right]$ are clearly observed.

of the Al multipole intensity is more gradual if only the inelastic scattering is considered, and the enhanced attenuation is caused by the damping of the $\mathrm{Al}$ multipole plasmon by presence of the adlayer charge density.

In order to understand the adlayer charge density-induced damping on another adlayer, we have studied the suppression of Na collective excitations as a function thickness of $\mathrm{K}$ adlayers deposited on a 2-ML-thick $\mathrm{Na}$ adlayer on $\mathrm{Al}(111)$. The $\mathrm{Na}$ double layer sustains both the bulklike and the multipole plasmon in contrast to the bulk Al substrate. Thus, in this case, the effect of adlayer $(\mathrm{K})$ growth on both excitations can be studied. Figure 8 shows a set of AERPY spectra with successive $\mathrm{K}$ deposition on a 2-ML $\mathrm{Na}$ layer grown on $\mathrm{Al}(111)$. The Na layer exhibits well defined Na-related multipole and bulk-plasmon excitations. For 0.4-ML K coverage, although no extra features develop, both the $\mathrm{Na}$ bulk and multipole plasmons are suppressed in intensity by about $30 \%$. The Na plasmons do not shift in frequency with $\mathrm{K}$ adlayer growth, as in the case of the $\mathrm{Al}$ multipole plasmon on alkali-metal deposition. By 0.8-ML $\mathrm{K}$ deposition, the AERPY line shape is drastically changed with further suppression of $\mathrm{Na}$ plasmons, and a broad intensity below $4 \mathrm{eV}$ appears, corresponding to the $\mathrm{K}$ mixed plasmon response. At 1.2 ML's, the trend of Na plasmon attenuation and enhancement of $\mathrm{K}$ related signal continues. At 2 ML's, the Na plasmons are almost fully suppressed, while the $\mathrm{K}$ multipole and bulk-plasmon modes are clearly observed at 3.2 and $3.8 \mathrm{eV}$, respectively. The observation that only two monolayers of $\mathrm{K}$ almost completely suppress the Na plasmons cannot be explained only by the inelastic scattering of photoelectrons, which at this K coverage would account for only $13 \%$ intensity attenuation. This is because the mean free path increases sharply as the electron kinetic energy decreases below $10 \mathrm{eV}$ and can be as large as 15 ML's (which is about $55 \AA$ for the $\mathrm{K}$ adlayer) in the photon energy range of the $\mathrm{Na}$ collective excitations $(\sim 6 \mathrm{eV}) .{ }^{51}$ Hence, if the inelastic scattering channel alone was responsible for the plasmon intensity decay with adlayer growth, the $\mathrm{Na}$ plasmon attenuation rate due to $\mathrm{K}$ adlayers would be much less than the $\mathrm{Al}$ plasmon attenu- ation by alkali-metal adlayers. It should be noted that the $\mathrm{Na}$ bulk plasmon is attenuated in a similar way as the $\mathrm{Na}$ multipole plasmon, showing that these modes are not decoupled.

\section{Influence of adatom induced surface structures on collective excitations}

Creating intermediate surface charge-density profiles through the formation of complicated surface structures may be used to study the dependence of collective excitations on the charge profile. The $\operatorname{Al}(111)-(\sqrt{3} \times \sqrt{3}) R 30^{\circ}: \mathrm{K}$ surface structure is unique in this sense because a significant change in the surface structure and hence the electron charge-density profile at the surface is expected by just changing the substrate temperature. ${ }^{41,43}$ Since the surface collective excitations depend critically on charge-density profile, interesting modifications in the AERPY spectra may be expected in this system. At low coverages $(\Theta \leqslant 0.15)$, the adlayer atoms occupy the threefold hollow sites and are uniformly distributed due to adsorbate-adsorbate repulsion. For increasing coverage, island formation occurs and the $\operatorname{Al}(111)-(\sqrt{3}$ $\times \sqrt{3}) R 30^{\circ}: \mathrm{K}$ structure forms at $\Theta=0.33$. $^{41,43,44}$ When the substrate is held at a temperature below $220 \mathrm{~K}$, nonactivated adsorption takes place and the $\mathrm{K}$ atoms occupy the on-top sites. These on-top sites become energetically favorable because of the accompanying relaxation of the $\mathrm{Al}$ atoms in the first substrate layer. The $\mathrm{Al}$ atom beneath the on-top $\mathrm{K}$ atom moves down while the atoms between the on-top $\mathrm{K}$ atoms move upward, relative to the unrelaxed surface (Fig. 9). ${ }^{43}$ This structure is henceforth referred to as the on-top $\sqrt{3}$ structure. At room temperature, activated adsorption occurs and the $\mathrm{K}$ atoms occupy the substitutional sites, although the surface symmetry remains the same $(\sqrt{3})$. In this substitutional structure, each $\mathrm{K}$ adatom replaces one $\mathrm{Al}$ atom from the substrate first layer (see Fig. 9). However, because the K atom is larger than the $\mathrm{Al}$ atom, it sits more towards vacuum (higher $z$ ) than the substrate atom did. This surface structure is henceforth referred to as the substitutional $\sqrt{3}$ structure. The on-top $\sqrt{3}$ structure is metastable and once the adatom is in the substitutional site, even if the temperature is lowered it will remain there.

In the case of $\mathrm{Na}$, deposition at room temperature leads to the formation of a $\operatorname{Al}(111)-(\sqrt{3} \times \sqrt{3}) R 30^{\circ}: \mathrm{Na}$ phase at a coverage of $\Theta=0.33$. Surface extended $x$-ray-absorption fine structure (EXAFS) as well as DFT-LDA calculations show that the $\mathrm{Na}$ atoms in this structure occupy sixfold coordinated substitutional sites. ${ }^{38}$ An on-top $\sqrt{3}$ structure for $\mathrm{Na} /$ $\mathrm{Al}(111)$ is not reported in the literature. However, the interesting aspect of this system is that around $\Theta=0.5$, a $(2 \times 2)$ structure is formed. ${ }^{37,40,42}$ This is referred to as a surface alloy phase consisting of four layers ( $\mathrm{Na}-\mathrm{Al}-\mathrm{Na}-\mathrm{Al})$, where the top three layers form a Na-Al-Na sandwich on the reconstructed Al fourth layer (see Fig. 1 of Ref. 40).

For $\mathrm{Al}(111)-(\sqrt{3} \times \sqrt{3}) R 30^{\circ}: \mathrm{K}$, an interesting difference between the AERPY spectra for the substitutional and the on-top $\sqrt{3}$ structures is the strong increase in intensity in the energy region of the $\mathrm{K}$ plasmons for the latter structure (Fig. 9). The spectrum smoothly increases in intensity from $4 \mathrm{eV}$ until the work-function cutoff, but no distinct peaks corre- 


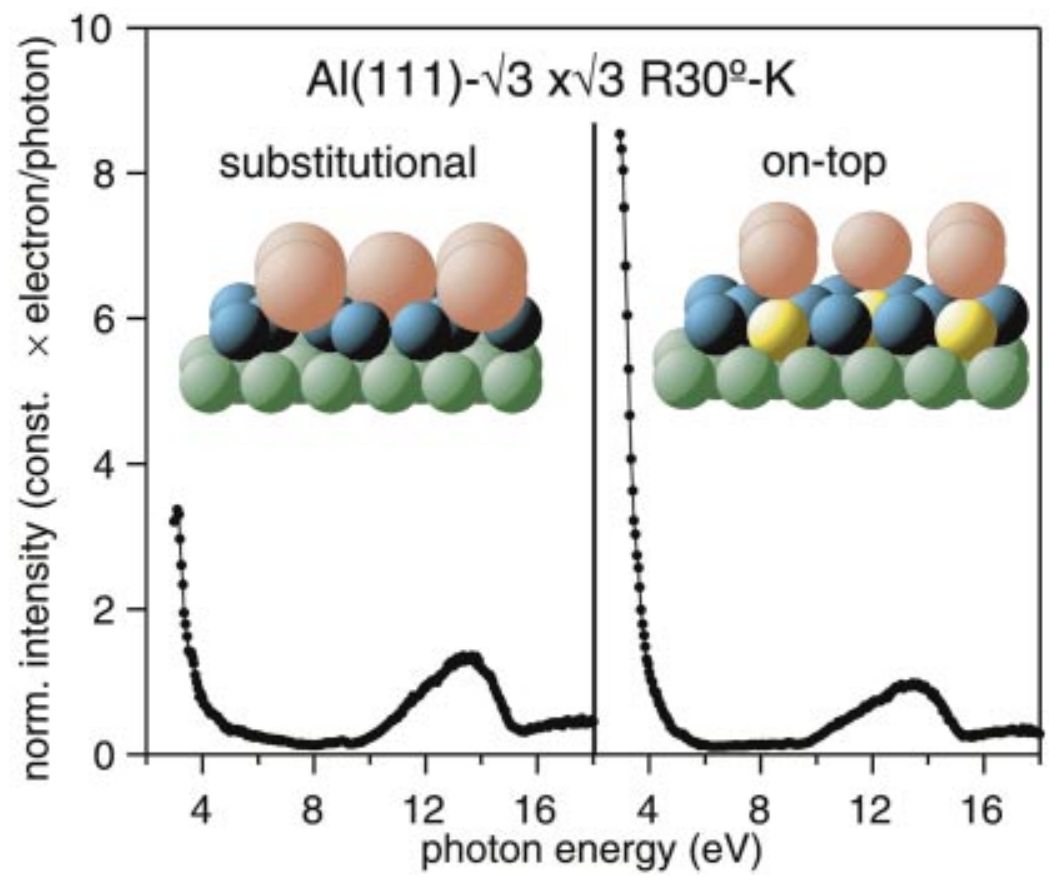

FIG. 9. (Color) Comparison of the photoyield spectra between the substitutional and the on-top $\sqrt{3}$ structures in $\mathrm{Al}(111)-(\sqrt{3} \times \sqrt{3}) R 30^{\circ}: \mathrm{K}$, exhibiting a strong increase in intensity in the ontop structure in the $\mathrm{K}$ plasmon region. A perspective view of both the structures are shown as inset. In the on-top structure, the $\mathrm{Al}$ atoms (yellow) below the $\mathrm{K}$ (red) atoms are displaced towards the bulk and the $\mathrm{Al}$ (blue) atoms between the $\mathrm{K}$ atoms are displaced towards vacuum. In the substitutional structure, the $\mathrm{K}$ (red) atoms kick out the $\mathrm{Al}$ atoms beneath them and occupy their positions. The second Al layer is shown in green to distinguish from the first Al layer. sponding to the $\mathrm{K}$ multipole or the bulklike plasmon are observed. A comparison between the $\sqrt{3}$ structures and clean $\mathrm{Al}(111)$ shows that the $\mathrm{Al}$ multipole plasmon is substantially reduced in intensity in the $\sqrt{3}$ structures, though its frequency remains unchanged. Between the substitutional and the on-top $\sqrt{3}$ structures, the Al multipole plasmon is attenuated in intensity by about $23 \%$ in the latter.

Since the origin of the multipole plasmon mode is intimately related to the nature of the ground-state chargedensity profile at the surface, we have calculated the laterally averaged charge-density profiles for clean $\mathrm{Al}(111)$ and the $\sqrt{3}$ structures (Fig. 10). These theoretical calculations are based on the self-consistent ab initio total-energy DFT-LDA method, the details of the calculation are described elsewhere. $^{41}$

The clean Al surface (dotted curve) shows a charge spillage at the surface which is generally observed for clean metals. In fact, the origin of the Al multipole plasmon is related to this smoothly varying density profile at the surface. ${ }^{1}$ Compared to the clean surface, the substituted $\sqrt{3}$ structure exhibits a clear step (about $67 \%$ of the total charge density) at the position of the first substrate layer. The origin of this step is not difficult to understand since, as discussed earlier, in this structure one-third of the $\mathrm{Al}$ atoms of the first substrate layer are replaced by $\mathrm{K}$ atoms. The electron density of $\mathrm{K}$, which is less than Al by about a factor of 13, hardly contributes in this region. Instead, the K-related charge enhances the diffuse tail of the substrate charge density (Fig. 10). The on-top $\sqrt{3}$ structure does not exhibit the step as in the substitutional structure because the $\mathrm{K}$ atoms do not disrupt the substrate first layer. The interesting difference in chargedensity profile between the two $\sqrt{3}$ structures is that the ontop geometry has a stronger and more diffuse charge-density tail related to the $\mathrm{K}$ adlayer (see the inset of Fig. 10). The difference between the two density profiles (dot-dashed curve in the inset of Fig. 10) clearly shows the excess charge in the on-top $\sqrt{3}$ structure. The reason for this difference is related to the structural difference between the two as shown in Fig. 9. In fact, this difference of the charge-density profile explains the enhanced intensity in the AERPY spectrum in the $\mathrm{K}$ plasmon region for the on-top structure compared to the substitutional structure. The $\sqrt{3}$ structure with $\Theta=0.33$ is similar in coverage to $1-\mathrm{ML} \mathrm{K} / \mathrm{Al}(111)(\Theta=0.45)$ and hence the 1-ML spectrum in Fig. 2 is similar to the on-top spectrum

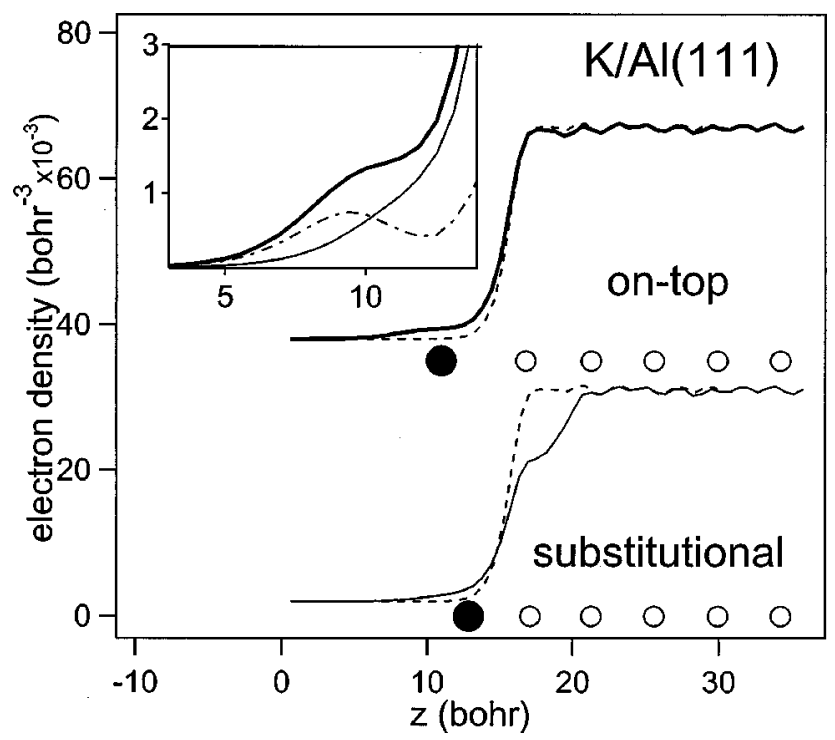

FIG. 10. Calculated electron charge-density profile for the ontop (thick line) and the substituted (thin line) $\sqrt{3}$ structure in $\mathrm{Al}(111)-(\sqrt{3} \times \sqrt{3}) R 30^{\circ}: \mathrm{K}$. The profiles (solid line) are compared to that of clean $\mathrm{Al}(111)$ (dashed curve) for both the structures. The positions of the $\mathrm{K}$ (filled circle) and the $\mathrm{Al}$ (empty circle) atoms are shown. The inset shows the vacuum-adlayer region in an expanded scale. The dot-dashed curve in the inset corresponds to the excess charge in the on-top structure in the diffuse tail of the chargedensity profile. 


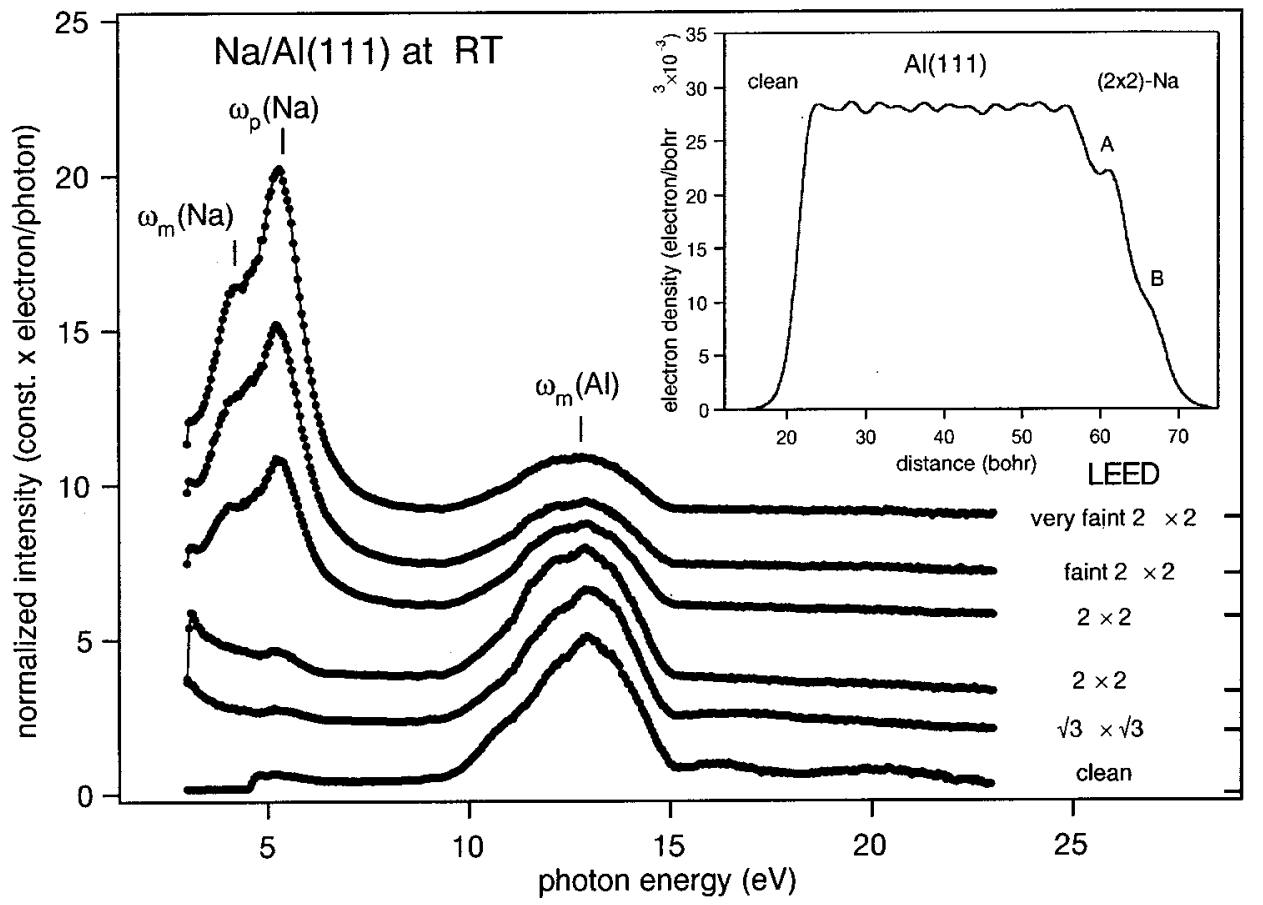

FIG. 11. AERPY spectra for different surface structures on $\mathrm{Na} / \mathrm{Al}(111)$ including the $\mathrm{Al}(111)$ $(2 \times 2)$ :Na surface alloy phase. The inset shows the laterally averaged charge-density profile for the $\mathrm{Al}(111)$ (left side) and the $(2 \times 2)$ surface alloy (right side), calculated using $a b$ initio DFT-LDA method. in Fig. 9. The intensity enhancement around $1 \mathrm{ML}$ is due to the broadened adlayer related bulklike and the multipole plasmon modes which are mixed together and redshifted. These modes are not separately observed because the adlayer charge-density plateau is not wide enough. However, for less than 2-ML coverage, with increasing diffusivity and strength of the K-related charge-density profile, an increase in intensity of the mixed plasmon mode is observed. This is the reason for the enhancement of the intensity in the $\mathrm{K}$ plasmon region in the on-top structure compared to substitutional $\sqrt{3}$ structure. The attenuation of the Al multipole plasmon intensity in the $\sqrt{3}$ structures can be related to the adlayer-induced damping, as discussed in the previous section.

A significant modification of the surface charge density is expected for the $(2 \times 2)$ surface alloy phase in $\mathrm{Na} / \mathrm{Al}(111)$. Hence the AERPY spectra for the different surface structures of $\mathrm{Na} / \mathrm{Al}(111)$ have been studied and the results are shown in Fig. 11. An important observation is the absence of a shift in frequency of the Al multipole plasmon upon surface alloy formation. A similar result was obtained for the substitutional $\mathrm{Al}(100)-c(2 \times 2): \mathrm{Na}$ surface alloy, where every second $\mathrm{Al}$ atom in the top layer is displaced by a $\mathrm{Na}$ atom. ${ }^{16}$ No such shift was observed for the $\sqrt{3}$ structures of $\mathrm{K}, \mathrm{Na}$, and $\mathrm{Li}$ either. Thus, for different alkali-metal adlayer-induced surface structures, the $\mathrm{Al}$ multipole plasmon frequency is unchanged. This finding is surprising because the alkali-metal electron density is much less than that of $\mathrm{Al}$ and a uniform dilution of the $\mathrm{Al}$ electron density by the alkali metals is expected to reduce the plasmon frequencies. In a recent TDLDA calculation on a rumpled $\mathrm{Al}(100)$ surface (with no $\mathrm{Na}$ atoms present) which is intended to represent the $\mathrm{Al}(100)-(2 \times 2)-\mathrm{Na}$ surface alloy, a redshift of about $2 \mathrm{eV}$ as well as a substantial intensity enhancement of the Al multipole plasmon was obtained. ${ }^{36}$ On the contrary, the present experimental data show neither any frequency shift nor any intensity enhancement of the Al multipole plasmon for different surface structures. The most plausible reason for the absence of a redshift of the Al multipole plasmon comes from the DFT-LDA calculations of the ground-state electronic structure. These calculations show that the Na-derived bands in the $(2 \times 2)$ surface alloy phase are empty while the Al-derived bands are filled and more stabilized by surface alloy formation. ${ }^{40,53}$ Thus the simplified jellium picture of charge dilution by surface alloy formation does not hold and the excess electron due to $\mathrm{Na}$ deposition essentially fills up the $\mathrm{Al}$ band. The Al multipole plasmon for the clean surface thus remains essentially unaffected by surface alloying.

The laterally averaged ground-state charge-density profiles for the clean and the $(2 \times 2)$-Na structure based on selfconsistent $a b$ initio total-energy DFT-LDA calculations are shown in the inset of Fig. 11. Details of the calculations can be found in Ref. 40 . The $(2 \times 2)-\mathrm{Na}$ (right side profile in the inset of Fig. 11) exhibits a kink (A) and a narrow step (B) at about 70 and $30 \%$ of the total charge density, respectively. Clean $\mathrm{Al}(111)$, on the other hand, exhibits a smoothly decreasing charge density (left side profile in the inset of Fig. 9).

No feature corresponding to the charge-density step (Fig. 10) in substitutional $\sqrt{3}$ structure of $\mathrm{K} / \mathrm{Al}(111)$ is observed (Fig. 9). Similarly, for the step features (A and B) in the $(2 \times 2)-\mathrm{Na}$ structure no new feature is observed in the AERPY spectrum (Fig. 11). Theoretical ${ }^{32,33}$ and present experimental data on smooth alkali-metal adlayers show that a sufficiently wide charge-density plateau (2 ML's, i.e., 5-6 A) is required to set up the collective modes corresponding to the charge density of the plateau. ${ }^{32,33,16}$ This ensures that the substrate-adlayer and the adlayer-vacuum interfaces are clearly separated. The steps observed in the above surface structures are not diffuse or wide enough to sustain a separate multipole plasmon mode at lower frequency. 
The intensity of the $\mathrm{Al}$ multipole plasmon remains mostly unchanged up to the $(2 \times 2)$ coverage in $\mathrm{Na} / \mathrm{Al}(111)$ (third spectrum from the bottom in Fig. 11). The increase in intensity below $6 \mathrm{eV}$ in the $(2 \times 2)$ structure can be related to $\mathrm{Na}$ mixed plasmon modes, as in case of $\mathrm{K}$. However, on further $\mathrm{Na}$ deposition, the $(2 \times 2)$ spots get fainter which indicates that the next layer of $\mathrm{Na}$ on the surface alloy phase does not grow in an ordered way. Only when this extra layer of $\mathrm{Na}$ starts growing on the $(2 \times 2)$ structure, one can observe the supression of the $\mathrm{Al}$ multipole plasmon intensity and the growth of the $\mathrm{Na}$ related multipole and the bulk plasmon clearly, as in the case of smooth adlayer growth at low temperature. This trend continues until the coverage where very faint $(2 \times 2)$ spots were observed, beyond which the AERPY spectra remained unchanged indicating that no further $\mathrm{Na}$ is sticking to the surface at room temperature. For the K/Al system, deposition of $\mathrm{K}$ on the on-top $\operatorname{Al}(111)-(\sqrt{3}$ $\times \sqrt{3}) R 30^{\circ}: \mathrm{K}$ structure also results in clearly separated multipole and bulklike plasmon modes as shown in Fig. 2 where the 1-ML coverage spectrum has the on-top $\sqrt{3}$ structure.

\section{CONCLUSION}

Our comprehensive study of the photon-excited collective modes in alkali-metal adlayers as well as the effect of the adlayers on the substrate $\mathrm{Al}(111)$ collective excitations shows that:

(1) Both the multipole and the standing-wave-like bulk plasmon modes are sustained by the $\mathrm{Na}$ and $\mathrm{K}$ adlayers, in good agreement with realistic as well as jellium based TDLDA calculations. ${ }^{32,33}$ Detailed comparison with theory (Fig. 3) shows, however, that (i) the FWHM is underestimated by TDLDA, ${ }^{33}$ which is probably because of electron scattering by defects and steps on a real surface, or damping due to interband transitions which are not considered in the theory. (ii) The plasmon frequency is over-estimated by 0.5 $\mathrm{eV}$ by the theory and this can be attributed to polarization of core electrons which tend to screen the plasmon oscillations.

(2) We find that the adlayer related plasmon modes appear not before the buildup of the second alkali-metal adlayer.

(3) Both bulk and multipole plasmon frequencies exhibit a blue shift up to about 5 ML's, and for thicker coverages a redshift is observed. While the redshift can be explained by the dispersion of the bulklike plasmon with wave vector $q$, the blue shift is attributed to the smooth density profiles at the interfaces.

(4) The frequency of the multipole plasmon is related to the bulk-plasmon frequency. For the different free-electron metals $(\mathrm{Na}, \mathrm{K}$, and $\mathrm{Al})$ studied here, our results show that the multipole plasmon occurs around $0.85 \omega_{p}$, independent of the electron density.

(5) The width of the adlayer bulk plasmon decreases while the multipole plasmon width increases with coverage.
(6) With increasing alkali-metal coverage, the bulk plasmon is suppressed in intensity due to decrease in coupling between the adlayer-vacuum and the substrate-adlayer interfaces.

The substrate $\mathrm{Al}$ multipole plasmon intensity is exponentially attenuated by adlayer growth, and this shown to be due to the damping of the substrate mode by the adlayer charge density. Damping of Na adlayer bulk and multipole plasmon by $\mathrm{K}$ adlayer growth reconfirms this effect. For $>2$-ML adlayer coverage, the substrate multipole plasmon, though diminished in intensity, is observed. This is probably because in the photon energy region where the $\mathrm{Al}$ multipole plasmon is excited, the $\mathrm{Na}(\mathrm{K})$ adlayers are transparent. The existence of the $\mathrm{Al}$ multipole plasmon even for 2 ML's of adlayer coverage indicates that the multipole plasmon can also exist at the substrate-adlayer interface.

In the case of the complicated adlayer induced surface structures, a relation between the diffusivity of the adlayerrelated charge-density profile and the intensity of the collective excitation modes is established. The Al multipole plasmon does not shift in frequency indicating the inadequacy of the simple concept of charge dilution in the surface alloys. The charge-density profile, calculated by the ab initio DFTLDA method, exhibits stepslike features at lower electron density which, however, are unable to sustain distinct collective excitations probably because they are not diffuse enough. For $\mathrm{Al}(111)-(\sqrt{3} \times \sqrt{3}) R 30^{\circ}: \mathrm{K}$, a strong increase in intensity in the energy region of the $\mathrm{K}$ plasmons is observed in the on-top structure (Fig. 9). This enhancement is attributed to a mixed plasmon mode. Growth of an extra alkali-metal layer on the surface structures $(2 \times 2$ and $\sqrt{3})$ results in the appearance of alkali-metal-related collective excitations and the attenuation of the $\mathrm{Al}$ multipole.

The existing TDLDA based theory in the frequency region of alkali-metal plasmons is in good agreement with the experimental results for smooth adlayers. However, although there are some experimental studies on the clean Al surface, hardly any theoretical results are available for the higher frequency Al multipole region addressing the mechanism of the substrate collective excitation damping as a function of the adlayer growth. For the adlayer induced surface structures on $\mathrm{Al}(111)$, there is no theoretical work which quantitatively predicts the nature of collective excitations. It is hoped that the present experimental results will simulate further theoretical studies in these directions.

\section{ACKNOWLEDGMENTS}

We thank A. Liebsch for his valuable comments on the manuscript. This work was supported by European Community grant no. CI1*-CT93-0059 (DG 12 HSMU), the Bundesministerium für Bildung und Forschung (grant 05622 OLA3), and the Indian (DST)-German (DLR) collaboration project (INI-038-99). 
${ }^{1}$ A. Liebsch, Elementary Excitation on Metal Surfaces (Plenum, New York, 1997).

${ }^{2}$ P.J. Feibelman, Prog. Surf. Sci. 12, 287 (1982).

${ }^{3}$ T. Miller, W.E. McMahon, and T.-C. Chiang, Phys. Rev. Lett. 77, 1167 (1996).

${ }^{4}$ E.D. Hansen, T. Miller, and T.-C. Chiang, Phys. Rev. Lett. 78, 2807 (1997).

${ }^{5}$ D. Claessen, S.-A․ Lindgren, L. Walldén, and T.-C. Chiang, Phys. Rev. Lett. 82, 1740 (1999).

${ }^{6}$ H.J. Levinson, E.W. Plummer, and P.J. Feibelman, Phys. Rev. Lett. 43, 952 (1979).

${ }^{7}$ K.D. Tsuei, E.W. Plummer, A. Liebsch, K. Kempa, and P. Bakshi, Phys. Rev. Lett. 64, 44 (1990).

${ }^{8}$ S.R. Barman, P. Häberle, and K. Horn, Phys. Rev. B 58, R4285 (1998)

${ }^{9}$ H. Petersen and S.B.M. Hagström, Phys. Rev. Lett. 41, 1314 (1978).

${ }^{10}$ S.A. Flodström and J.G. Endriz, Phys. Rev. Lett. 31, 893 (1973).

${ }^{11}$ G. Jezequel, Phys. Rev. Lett. 45, 1963 (1980).

${ }^{12}$ J. Monin and G. Boutry, Phys. Rev. B 9, 1309 (1974).

${ }^{13}$ L. Walldén, Phys. Rev. Lett. 54, 943 (1985).

${ }^{14}$ B. Kim, E. W. Plummer, and A. Liesch (unpublished).

${ }^{15}$ S.R. Barman, K. Horn, P. Häberle, H. Ishida, and A. Liebsch, Phys. Rev. B 57, 6662 (1988).

${ }^{16}$ S.R. Barman, C. Stampfl, P. Häberle, and K. Horn, Phys. Rev. B 61, 12721 (2000).

${ }^{17}$ R.H. Ritchie, Phys. Rev. 106, 874 (1957).

${ }^{18}$ E.W. Plummer, K.-D. Tsuei, and B. Kim, Nucl. Instrum. Methods Phys. Res. B 96, 448 (1995), and references therein.

${ }^{19}$ E.W. Plummer, Solid State Commun. 84, 143 (1992).

${ }^{20}$ A. Bennett, Phys. Rev. B 1, 203 (1970).

${ }^{21}$ A. Eguiluz, S.C. Ying, and J.J. Quinn, Phys. Rev. B 11, 2118 (1975).

${ }^{22}$ J.E. Inglesfeld and E. Wikborg, J. Phys. F: Met. Phys. 5, 1706 (1975).

${ }^{23}$ C. Schwartz and W.L. Schaich, Phys. Rev. B 30, 1059 (1984).

${ }^{24}$ K. Kempa and R.R. Gerhardts, Solid State Commun. 53, 579 (1985).

${ }^{25}$ K.-D. Tsuei, E.W. Plummer, A. Liebsch, E. Pehlke, K. Kempa, and P. Bakshi, Surf. Sci. 247, 302 (1991).
${ }^{26}$ T. Lopez-Rios, in Spatial Dispersion in Solids and Plasmons (North-Holland, Amsterdam, 1992), p. 215, and references therein.

${ }^{27}$ A. Liebsch, Phys. Rev. B 36, 7378 (1987).

${ }^{28}$ J.W. Gudzuk, Phys. Rev. B 1, 1267 (1970).

${ }^{29}$ P.J. Feibelman, Phys. Rev. Lett. 30, 975 (1973).

${ }^{30}$ F. Forstmann and H. Stenschke, Phys. Rev. Lett. 38, 1365 (1977).

${ }^{31}$ A. Liebsch, Phys. Rev. B 40, 3421 (1989).

${ }^{32}$ A. Liebsch, Phys. Rev. Lett. 67, 2858 (1991).

${ }^{33}$ H. Ishida and A. Liebsch, Phys. Rev. B 45, 6171 (1992).

${ }^{34}$ A. Liebsch, G. Benemanskaya, and M. Lapuskin, Surf. Sci. 302, 303 (1994).

${ }^{35}$ W.L. Schaich, Phys. Rev. B 55, 9379 (1997).

${ }^{36}$ H. Ishida, and A. Liebsch, Phys. Rev. B 57, 12550 (1998).

${ }^{37}$ J. Burchhardt, M.M. Nielsen, D.L. Adams, E. Lundgren, J.N. Andersen, C. Stampfl, M. Scheffler, A. Schmalz, S. Aminpirooz, and J. Haase, Phys. Rev. Lett. 74, 1617 (1995).

${ }^{38}$ A. Schmalz, S. Aminpirooz, L. Becker, J. Haase, J. Neugebauer, M. Scheffler, D.R. Batchelor, D.L. Adams, and E. Bogh, Phys. Rev. Lett. 67, 2163 (1991).

${ }^{39}$ J.N. Andersen, M. Qvarford, R. Nyholm, J.F. van Acker, and E. Lundgren, Phys. Rev. Lett. 68, 94 (1992).

${ }^{40}$ C. Stampfl and M. Scheffler, Surf. Sci. Lett. 319, L23 (1994).

${ }^{41}$ C. Stampfl, J. Neugebauer, and M. Scheffler, Surf. Sci. 307-309, 8 (1994).

${ }^{42}$ A. Hohfeld and K. Horn, Surf. Sci. 211/212, 844 (1989).

${ }^{43}$ C. Stampfl, M. Scheffler, H. Over, J. Burchhardt, M. Nielsen, D.L. Adams, and W. Moritz, Phys. Rev. B 49, 4959 (1994).

${ }^{44}$ C. Stampfl and M. Scheffler, Surf. Rev. Lett. 2, 317 (1995).

${ }^{45}$ A.v. Felde, J. Sprösser-Prou, and J. Fink, Phys. Rev. B 40, 10181 (1989).

${ }^{46}$ K. Sturm, Solid State Commun. 48, 29 (1983).

${ }^{47}$ A.R. Melynk and M.J. Harrison, Phys. Rev. Lett. 21, 85 (1968).

${ }^{48}$ M. Anderegg, B. Feuerbacher, and B. Fitton, Phys. Rev. Lett. 27, 1565 (1971).

${ }^{49}$ A. Pimpale and B.V. Paranjape, Surf. Sci. 161, 69 (1985).

${ }^{50}$ F. Sauter, Z. Phys. 203, 488 (1967).

${ }^{51}$ M.P. Seah and W.A. Dench, Surf. Interface Anal. 1, 2 (1979).

${ }^{52}$ D.R. Penn, Phys. Rev. B 13, 5248 (1976).

${ }^{53}$ C. Stampfl, K. Kambe, R. Fasel, P. Aebi, and M. Scheffler, Phys. Rev. B 57, 15251 (1998). 